\title{
Development and Analysis of Simplified Control-oriented Models for a Group of Institutional Offices
}

\author{
Jayson Bursill $^{1}$, William O'Brien ${ }^{1}$, Ian Beausoleil-Morrison ${ }^{1}$ \\ ${ }^{1}$ Carleton University, Ottawa, Canada
}

\begin{abstract}
Most zone or room level control-oriented models are developed with opaque and varying workflows. The workflows vary depending on whether the models are white or black box. In this paper the focus is applied to inverse modelling. The contribution of this paper is the application of a simple prescribed workflow for generating inverse models of a group of offices that can be applied by those less experienced with inverse modelling. The goal of the models is to predict indoor air temperature at the next time step for each room with reasonable accuracy. Measurements were taken in 27 offices for a period of one month. These data were used to fit and validate office level control-oriented inverse models using the MATLAB System Identification Toolbox. The maximum mean absolute error (MAE) for any office over the two-week validation period was $0.31^{\circ} \mathrm{C}$ and the average MAE for all 27 offices was $0.15^{\circ} \mathrm{C}$
\end{abstract}

\section{Introduction}

Model-based predictive control (MPC) has demonstrated the potential to save up to $50 \%$ of heating and cooling energy in commercial buildings (Dong, O'Neill, \& Li, 2014). The quality and suitability of the underlying modelling is essential to achieve this potential. A balance between model accuracy and computational efficiency for scalability is necessary.

Control-oriented modelling is a critical component of zone-level MPC (B. Gunay, O'Brien, \& BeausoleilMorrison, 2016; H. Burak Gunay, Bursill, Huchuk, O'Brien, \& Beausoleil-Morrison, 2014). Modelling for zone-level MPC can vary in detail from white box software-based modelling (e.g., EnergyPlus (Zhao, Lam, Ydstie, \& Karaguzel, 2015)) to black box function-based modelling (e.g., state-space models (B. Gunay et al., 2016)). At the zone level in commercial buildings, black (or grey) box models have been found to provide a compromise between model accuracy and development time (H. Burak Gunay et al., 2014). Data storage limitations are no longer a major challenge for controloriented modelling due to the increase in building automation system (BAS) data storage seen in the mid2000s (Salsbury, 2005).

Several studies have investigated control-oriented inverse modelling using measured data. Gunay et al. (2016) applied a recursive approach (including an extended Kalman filter) to 16 offices in an institutional building to obtain control-oriented state-space models. Other studies have provided a workflow for MPC, but remain focused on the implementation of the algorithm itself (Froisy,
2006; Joe \& Karava, 2016). Despite the efforts of recent studies, the details of the workflow have remained complicated and difficult to implement for non-expert inverse modellers or unsuitable for state-space temperature modelling by focusing on extensive and often physical properties that are difficult to estimate.

The contribution of this paper is the application of a clear data-driven inverse modelling workflow to simplified models that is accessible to less experienced inverse modellers. In this study the workflow is applied to a group of actively utilized offices with a large suite of integrated sensors while describing the details of the data acquisition and cleaning process, something often overlooked. Previous studies have utilized detailed simulation as the source for inverse modelling data, where real disturbances cannot be measured (Candanedo \& Athienitis, 2011; H. Burak Gunay et al., 2014). Model form and node quantity is another important consideration (Kim \& Braun, 2014), where multi-node models were selected as a compromise between simplicity and accuracy.

This paper describes a standard workflow to generate control-oriented state-space models from the BAS data of an institutional building office block. This process is then applied to the 27 rooms introduced in this study, and the resulting model parameters and characteristics are discussed. Background and application (in the context of this study) are described in separate sub-sections of the modelling workflow methodology section. The resulting models can be used within zone level detailed MPC algorithms in office buildings with modern building automation systems and office-level sensing to reduce computation time while maintaining accuracy (when compared to detailed models).

\section{Modelling workflow methodology}

While it is acknowledged that many unique workflows are viable (and potentially easily implemented), the proposed workflow utilized in this study offers a transparent approach for a zone level control-oriented modelling case. The target audience is users less familiar with deriving inverse models. The limitations and assumptions of each step in the workflow will be elaborated following the list in Figure 1.

The following sections outline the requirements of each step and demonstrate the application of the workflow in an institutional building in Ottawa, Canada during the cooling season. The workflow and its iterative paths is provided in Figure 1. 


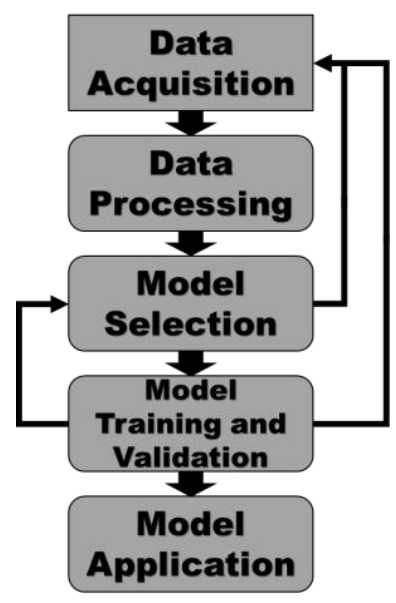

Figure 1: Modelling workflow flowchart

Data was acquired from 27 newly-constructed occupied offices. The offices serve as a living laboratory, in that the researchers designed the instrumentation, but do not control occupants' use of the space.

\section{Data acquisition - methodology}

Sensor data storage from the BAS that controls and monitors the studied offices was essential to developing control-oriented models. Without long-term data storage (sensor logs with more than one day of data) inverse modelling, operational analytics, and troubleshooting become difficult and qualitative. The volume of available data renders the selection of appropriate sensors for modelling a non-trivial task. This section is largely application based to provide context to the example and supplement the literature for data acquisition and collection. The following application focuses on describing the building studied and collecting bulk data that can be pared down after the model selection process is complete.

\section{Data acquisition - application}

In this study data was logged in an industrial computer for long term storage. The desired points from the BAS upload data to the industrial computer every four hours (with short term logs at the controller level that typically $\log$ for much longer than four hours but overwrite when full). Data can be stored for years on the industrial computer, and even longer in an adjacent cloud storage service.

Sensor data were collected from 27 rooms in the institutional building studied (façade shown in Figure 2). The initial data was eventually processed and reduced to only the required inputs for the proposed model (as determined by model selection). The building automation system was completed in the summer of 2018, therefore only roughly six months of data is available at the time of this study (three of which are from the cooling season). Models in this study were trained with two weeks of data, validated with the following two weeks, and the models were utilized in an MPC algorithm for the remaining weeks of the cooling season.
The external wall glazing ratio was calculated to be 0.3 for rooms with one of four walls being external and 0.8 for corner rooms.

The zone level cooling system consists of a variable air volume (VAV) terminal unit to regulate airflow that serves between one and four rooms. Airflow was driven by a fan at the air handling unit where air was initially cooled to a temperature that meets the highest zone demand for the floor. The airflow to each office was assumed to be an equal division of the zone airflow measured at the VAV box. The mechanical drawings show that each office is the same size, shape, and has the same diffuser location and orientation. Ducts from the VAV boxes to the diffusers would vary in geometry according to the number of rooms in the zone (e.g., one to four branches). When cooling is required, airflow is modulated via the VAV zone level air flow damper to allow the space to achieve a setpoint as defined by the occupant. The setpoint could be adjusted by the occupants of each room via the thermostat and could vary from $20^{\circ} \mathrm{C}$ to $25^{\circ} \mathrm{C}$. Most rooms maintained a setpoint between $21.5^{\circ} \mathrm{C}$ and $23.0^{\circ} \mathrm{C}$. The VAV box also provides the outdoor air supply requirement and is active on a schedule from 7:30AM to 7:00PM supplying an average of 1,700 $1 / \mathrm{s}$ to the entire office group when active (and no airflow when inactive). The floor level air handling unit operates 24 hours per day every day of the week because it supplied lab spaces as well and had an average supply air temperature of $14.1^{\circ} \mathrm{C}$. Due to the large amount of exhaust air from the lab spaces the outdoor air fraction was greater than 0.9 for most of the data acquisition period to maintain building pressurization.

Data was sampled at 5-minute intervals for analog inputs (e.g., temperature) and upon state change for binary inputs (e.g., window opening). Binary values from passive infrared occupancy sensors were utilized to infer thermal loads from occupants and equipment (e.g., lighting and plug in equipment) as suggested in the literature (B. Gunay et al., 2016). This was found to be acceptable when the model accuracy was assessed. Analog BAS readings were verified within $\pm 10 \%$ via independent spot measurements. All offices are oriented with the primary façade facing 30 degrees North of East and are equipped with motorized blinds with solar and visible transmittances of $3 \%$ and $4 \%$ respectively. Motorized blinds were automatically closed after 5:00PM and reopened at 7:30AM on weekdays and could be manually overridden by the occupant. A diagram of a typical office and the adjacent portion of the cooling system is provided in Figure 3, with a mapping of the sensor schedule in Table 1. The thermistors and resistance temperature detectors had bias errors of $0.2^{\circ} \mathrm{C}$, while the illuminance sensors and pressure transducers had errors of $2 \%$ and $1 \%$ full scale respectively (equating to 20 lux and 5 pascals).

\section{Data processing- methodology}

Data processing has a high degree of difficulty largely due to data tagging issues within most building automation systems. Consistency in naming of points (e.g., "VAV_FLOW..." means VAV flow) is uncommon and 
nomenclature can even vary within buildings and floors if multi-stage fit-ups and retrofits/renovations occur. Data tagging difficulties are a known issue and efforts are being made within ASHRAE and other international organizations to create standards for naming (Charpenay, Käbisch, Anicic, \& Kosch, 2015; Dibowski, Vass, Holub, \& Rojíček, 2016). Single fit-ups with a consistent control programming team are ideal cases for control point naming consistency and rapid data processing. Another important consideration when processing measured data for inverse modelling is the alignment of sampled data. Analog inputs measuring different quantities (e.g., airflow, temperature) are frequently logged at different rates to save space within room/zone level controllers. This necessitates either the discarding a large portion of the data or interpolation. The method that is more suitable is dependent on the rate of change of the input with a lower sample rate. These issues are compounded when aligning the time stamps of event-base data, which will likely occur outside of regular sample intervals. Resultantly, even the simple task of obtaining a complete data set with both continuous and event-based data can be a difficult task in some buildings. Event-based data can be attributed to the nearest continuous sample to the event (e.g., a state-change at 12:02AM can be mapped to a 12:00AM continuous sample). Attributing the eventbased data to continuous samples can lead to errors of event-based input run time of up to one whole sample interval (half of an interval on each side) and can propagate significantly if frequent cycling occurs. The conclusion of the data processing step is often indeterminate, and further lends to the cyclical nature of the proposed workflow.

\section{Data processing- application}

In the studied building, a consistent naming convention was utilized, and no renovations occurred prior to the study period because it was recently constructed (substantially completed in summer 2018). Event based data is the occupant state, inferred by the passive infrared sensor binary feedback. No sensors utilized in the applied modelling workflow were found to be faulty, and data processing was without complication (which is often not the case). Complications in data processing are common but can be mitigated with a consistent BAS programming and tagging approach during construction or retrofits as described in the data processing - background section.

\section{Model selection - methodology}

Model selection as presented in this paper refers to selecting the appropriate inputs for single node linear time-invariant (LTI) state-space models. While there are virtually limitless potential model types, single node LTI state-space models were selected in this paper because they were found to be appropriate for MPC applications and both robust and simple (Bursill, O'Brien, \& Beausoleil-Morrison, 2018). The scope of model selection can become very large when many different types of models are evaluated and compared.

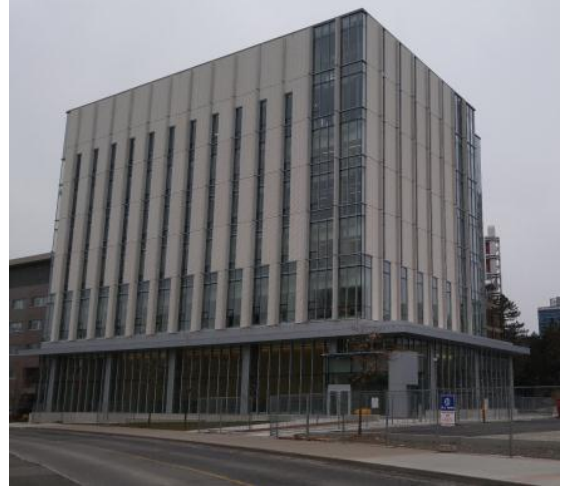

Figure 2: Building façade of inverse modelled rooms.

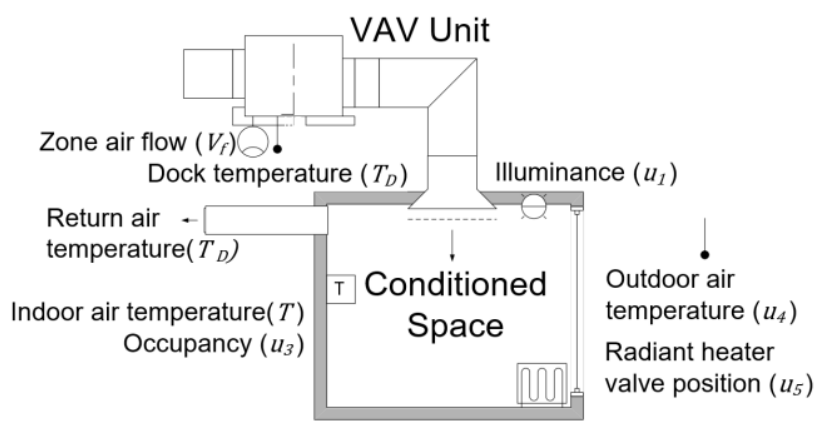

Figure 3: Diagram of conditioned space with HVAC system and sensors.

Table 1: Sensor schedule

\begin{tabular}{|c|c|}
\hline Measured Quantity & Sensor \\
\hline Zone air flow $\left(V_{f}\right)$ & Differential pressure transducer \\
\hline $\begin{array}{c}\text { Indoor air temperature } \\
(T)\end{array}$ & $10 \mathrm{kOhm}$ thermistor \\
\hline $\begin{array}{l}\text { VAV box outlet } \\
\text { temperature }\left(T_{D}\right)\end{array}$ & $\begin{array}{c}\text { 4-20 mA Resistance temperature } \\
\text { detector }\end{array}$ \\
\hline Illuminance $\left(u_{1}\right)$ & Photodiode \\
\hline Occupancy $\left(u_{3}\right)$ & Passive infrared \\
\hline $\begin{array}{c}\text { Outdoor air temperature } \\
\left(u_{4}\right)\end{array}$ & $10 \mathrm{kOhm}$ thermistor \\
\hline $\begin{array}{c}\text { Radiant heater valve } \\
\text { position }\left(u_{5}\right)\end{array}$ & Output feedback \\
\hline
\end{tabular}

This paper will focus on applying the workflow to a statespace model that is an analogue of a typical thermal resistance and capacitance model (Gouda, Danaher, \& Underwood, 2002). In practice this workflow could be applied for different model types, but it is assumed a general model type is pre-selected. The model selection process is a topic of many publication in the literature, where the following are merely a starting point: (Athienitis, 1994; Bacher \& Madsen, 2011; Gouda et al., 2002; H. Burak Gunay et al., 2014; Joe \& Karava, 2016). Figure 4 shows a typical first-order grey box model of room indoor air temperature that includes aggregate thermal resistance $\mathrm{R}$, aggregate thermal capacitance $\mathrm{C}$, outdoor air temperature $\mathrm{T}_{\mathrm{o}}$, and total heat input $\mathrm{Q}_{\text {tot }}$. The model in Figure 4 is a good starting point for the proposed state-space models. Total heat input can be replaced by 
individual energy flows and inferred as a proxy of their respective input values in the state-space model form (e.g., $u_{1}$ to $u_{5}$ in Table 1 ). Cases where a simplified statespace model are not applicable may exist where extremely non-linear conditions occur. It is assumed that the same procedure can be implemented with modifications to the model form and volume of required data.

Several approaches for inverse model input selection exist, including (but not limited to): forward selection (Bacher \& Madsen, 2011), back propagation, and principle component analysis (Yang, Rivard, \& Zmeureanu, 2005). The three approaches presented are described in detail in the referenced literature and provide approaches for simple models with a small number of inputs, complicated black box models, and models with many inputs respectively. Forward selection (the simplest approach presented) relies on comparing the error of model forms with different numbers of inputs to determine the best trade-off between complexity and accuracy. Back propagation is mostly applied to artificial neural network models to quickly pare down many hidden layers simultaneously. Principle component analysis utilizes clustering and linear correlations to determine the usefulness of model inputs.

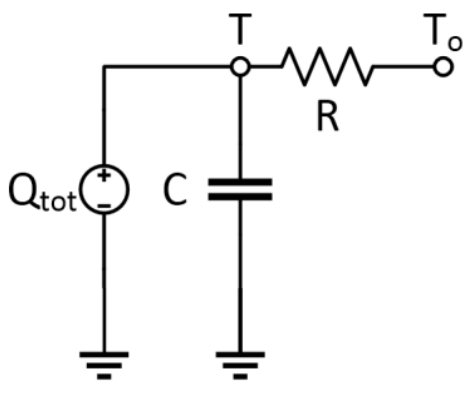

Figure 4: Diagram of typical RC grey box model

\section{Model selection - application}

For the state-space IAT models in this study, higher order models add complexity and increase the programming requirements within MPC algorithms and BAS databases. Time-variant modelling (where parameters update over time to improve prediction accuracy) also adds complexity and the requirement of determining an appropriate retraining rate or the addition of a recursive approach.

Identifying and stating assumptions of the proposed model is central to the model selection process. Many are described in the Data acquisition section, but further assumptions regarding the model itself are provided here. Perhaps the most important assumption is that inputs can be linearized within the range of conditions observed in this study for the linear models. It is also assumed that the lux sensor can be used as a proxy for solar radiation. This was confirmed in the literature (Bursill et al., 2018; B. Gunay et al., 2016).

Assumed room properties included negligible air side thermal short-circuiting and steady-state cooling between sample periods. Heat transfer to adjacent conditioned spaces is minimal. All thermal storage was attributed to the single node room air temperature model. Perfectly mixed air is assumed in this model.

Indoor air temperature (IAT) was selected as the predicted state of the state-space model because it can be directly compared to the desired setpoint for each space to estimate if heating or cooling is required (and is a requirement of many MPC algorithms). In this study a combination of forward selection and principle component analysis was utilized to retain simplicity and handle the large number of inputs. Linear correlations between the predicted value (IAT at the next time step) and inputs at the current time step were evaluated to determine appropriate model inputs. Only physically sensible inputs with a linear correlation coefficient of greater than 0.2 were selected. The threshold of 0.2 was chosen because a gap of 0.08 was present between this threshold and the next tier of inputs, which was significantly larger than other gaps between correlation coefficients in the data used in this study. Additionally, inputs that were strongly linearly related to inputs already selected were removed to promote orthogonality (i.e., the absence of correlation between inputs). Pure forward selection was not utilized because there were between 80 and 115 inputs for each room in this study. A limitation of this approach is that poor linear correlations could indicate that an input is a poor indicator of IAT change or that the linear model is inappropriate. The latter case is mitigated through cross-validation of the proposed model in the following section.

Linear correlation analysis of all sensors and variables within the BAS controller databases using the previously mentioned criteria yielded the selected model form to predict IAT. The correlation analysis was limited to the linear correlation of individual inputs with IAT prediction for one time step and did not look at the effects of combining multiple inputs simultaneously or compounded predictions over multiple time steps. Equation 1 presents the general form of the proposed state-space model where $T$ is the indoor air temperature, $t$ is the time index, and $A$ and $B$ are matrices of constants generated from fitting the model to the data. Equation 2 utilizes air flow rate $\left(V_{f}\right)$, IAT $(T)$, and VAV box outlet temperature $\left(T_{D}\right)$ to estimate a cooling energy proxy $u_{2}(t)$ assuming constant air properties and neglecting latent cooling. The previously listed inputs were selected as a result of the linear correlation study. Sensible cooling loads were found to be dominant, where sensible heat fractions of 0.6 to 0.9 were observed at the coil of the air handling unit over the cooling season for the zones served. Latent cooling, though not unsubstantial, was not considered in this study due to the high observed inaccuracy and bias error of the installed relative humidity sensors and could be the focus of future work in the presence of improved humidity sensing. Equation 3 presents the form where each element of the matrix $B$ is mapped to inputs from Table 1 and Equation 2 for the single node case. Only the first four inputs were used in the cooling season model. The ellipse in Equation 3 
represents the location to add sensors for different model forms or to include heating terminal units in the heating season. Equation 3 can be used to describe the model in Figure 5, a state-space adaptation of the model in Figure 4.

$$
\begin{gathered}
T(t+1)=A T(t)+B u(t) \\
u_{2}(t)=V_{f}(t)\left(T(t)-T_{D}(t)\right) \\
T(t+1)=A T(t)+B_{1} u_{1}(t)+B_{2} u_{2}(t)+B_{3} u_{3}(t) \\
+B_{4} u_{4}(t)+\cdots
\end{gathered}
$$

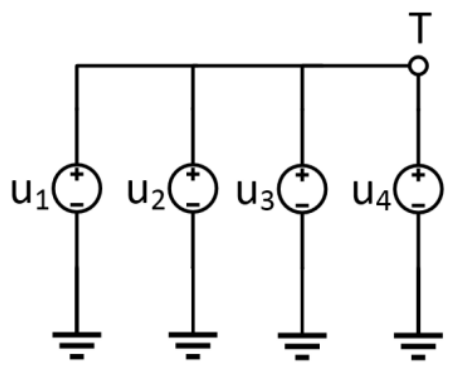

Figure 5: Diagram of selected state-space model

\section{Model training and validation - methodology}

The selected model form will have a large impact on which software and techniques are utilized for training. MATLAB offers a large array of training and analysis functions for different model forms, but large functional libraries for inverse modelling are also present in Python and other languages (Ljung, 1995). For this study MATLAB was selected for its training functions due to ease of implementation.

Model cross-validation is one of the simpler tasks within the proposed workflow, where the proposed model is applied to a portion of the collected data. In this case half of the data is used for validation, but different distributions of training and validation data may be used depending on the application (Shahin, Maier, \& Jaksa, 2004). A model is considered valid if the error metric, mean absolute error in this case (MAE), is below the bias error of the sensor obtaining the measurement.

\section{Model training and validation - application}

Sample intervals and time steps of 30 minutes were selected because most continuous BAS data is monitored at this frequency or higher (or only needs to be interpolated once from hourly data). The building studied had a sample interval of five minutes, but this is acknowledged to be uncommon in most existing commercial buildings. To adjust from five to 30 minute sample intervals only every sixth sample from the initial data was used. In previous analysis it was verified that model stability was retained at five minute time steps and accuracy was increased. The shorter time step also increases the computational requirements for MPC exponentially due to square matrix operation requirements (Wang, 2009). Thus, a time step of 30 minutes is a compromise of accuracy and efficiency in the room and zone level control-oriented modelling case for a three hour prediction horizon.

One month of sample data was divided equally into training and validation sets. The MATLAB System Identification Toolbox ssest function was used to obtain the matrices $A$ and $B$ of Equation 1 for each office from the sample data using the subspace method (Zhou, Pierre, $\&$ Hauer, 2006). Training was executed with two weeks of measured data from IAT and the inputs $u_{1}, u_{2}, u_{3}$, and $u_{4}$ from Table 1 and Equation 2.

A static value of bias error was chosen from the highest observed flow rate and temperature differential to encompass a conservative estimation of uncertainty. Input bias errors were obtained from catalogue information, where the bias error of the air temperatures was $0.2^{\circ} \mathrm{C}$. The precision error was found to be half of the bias error for the temperature sensors $\left(0.1^{\circ} \mathrm{C}\right)$.

One month of data split into two equal two week portions for training and validation was found to be suitable for model preparation, and the literature suggests that a week for each is suitable (B. Gunay et al., 2016).

\section{Model application}

Once an appropriate model has been selected, trained, and validated, it must be utilized in the algorithm it was designed for. This is the most open-ended portion of the proposed workflow and is on the edge of the scope of this paper. Some notable applications of the resulting indoor air temperature inverse model (or similar models) are:

- Embedded MPC feedback (e.g., predicting the future time step energy balance for the room).

- Predictive information for occupant engagement (e.g., visible feedback of future office thermal conditions to the occupant).

- Fault detection of anomalies such as incorrect terminal unit operation or envelope degradation (e.g., sensor feedback suggests the IAT should increase but it does not).

\section{Results and Discussion}

The resulting models from the workflow for the cooling season and their characteristics are presented in this section. A description of the parameters is provided in Table 2. Parameter values and the MAE are reported. Average, mean, maximum, minimum, and the coefficient of variation $(\mathrm{CV})$ as defined in Equation 4 are reported in Table 3 for the model form shown in Equation 3 and Figure 5.

Coefficient of variation is a useful metric to determine the consistency of estimated values when a typical value is not present in the literature for comparison (as is often the case with unique inverse model forms). While the distribution of the model parameters may not necessarily be Gaussian, the CV provides insight into which values obtained from inverse modelling are more consistent within the sample rooms. The input parameters that varied the most were related to cooling energy and outdoor air temperature. The high variation of the cooling energy proxy parameter (B2) and outdoor air parameter (B4) suggests that the rooms modelled react diversely to 
cooling and outdoor air conditions. Diversity of B2 could be attributed to differences in zoning (zones varied from one to four rooms per VAV unit), while B4 variation could be attributed to window usage (as confirmed by window data). The airflow was assumed to be divided equally between the rooms in each zone in the model, which could also be a source of error in both the proxy and the parameters. However, the inferences of these parameter variations are anecdotal, and the linear model parameters are not an absolute reference of relative room properties.

$$
C V=\frac{\text { Standard Deviation }}{\text { Average }}
$$

It is notable that the rooms with the highest MAE had significantly higher glazing ratios than the majority of the rooms ( 0.8 and 0.3 respectively). The high MAE of these rooms suggests that spaces with high glazing ratios are less suitable for linear parameter state-space modelling due to the impact of solar radiation on the indoor air temperature. In these models, solar radiation is inferred using illuminance sensors as a proxy, and solar radiation has a non-linear relationship with IAT. The effect of the two previously mentioned sources of error becomes significant when the glazing ratio is high and solar radiation becomes a dominant source of heat transfer for the room. Error could be mitigated in this case with more model nodes and potentially adding a nonlinear relationship with the solar inputs. Both suggested solutions require a significant increase in model complexity and computational time.

The use of a unified model form for a subset of controlled rooms is a boon to scalability. Model and algorithm scalability are often cited as limitations of state-of-the-art MPC implementations. A singular model form (albeit with differing parameter values) reduces scaling complications related to commercial adoption. While the corner rooms had different glazing ratios, additional exterior walls, and higher model MAE, the resulting error was still comparable to the rooms more suited to the selected model form. In future work the model selection step in the workflow could include dividing the set of modelled rooms by envelope and orientation characteristics into different model categories. Such classification has been previously examined in the literature and found to be appropriate for commercial buildings (Shi \& O’Brien, 2018).

Table 2: Parameter descriptions

\begin{tabular}{|c|c|}
\hline Parameter & Description \\
\hline A & Relation to IAT of previous time step \\
\hline B1 & Relation to illuminance (as a solar proxy) \\
\hline B2 & Relation to VAV cooling \\
\hline B3 & Relation to outdoor ait temperature \\
\hline B4 & Relation to occupancy \\
\hline
\end{tabular}

Table 3: Model parameter values for the cooling season. *indicates window to wall ratios $>0.6$.

\begin{tabular}{|c|c|c|c|c|c|c|}
\hline Room & $\begin{array}{c}\text { IAT } \\
\text { MAE } \\
\left({ }^{\circ} \mathbf{C}\right) \\
\end{array}$ & $\begin{array}{c}\text { A } \\
\text { (no unit) }\end{array}$ & $\begin{array}{c}\text { B1 } \\
\left({ }^{\circ} \mathrm{C} / \mathrm{lux}\right)\end{array}$ & $\begin{array}{c}\text { B2 } \\
\text { (s/L) }\end{array}$ & $\begin{array}{l}\text { B3 } \\
\left({ }^{\circ} \mathrm{C}\right)\end{array}$ & $\begin{array}{c}\text { B4 } \\
\text { (no unit) }\end{array}$ \\
\hline $1 *$ & 0.247 & 0.989 & 3.2E-4 & $2.4 \mathrm{E}-4$ & $1.3 \mathrm{E}-2$ & -0.063 \\
\hline $2 *$ & 0.273 & 1.003 & 7.1E-4 & $6.5 \mathrm{E}-4$ & $-4.1 \mathrm{E}-3$ & -0.094 \\
\hline 3 & 0.154 & 0.994 & $7.5 \mathrm{E}-4$ & $2.3 \mathrm{E}-4$ & $6.6 \mathrm{E}-3$ & -0.197 \\
\hline 4 & 0.073 & 0.996 & $5.9 \mathrm{E}-4$ & $1.7 \mathrm{E}-4$ & $3.9 \mathrm{E}-3$ & -0.046 \\
\hline 5 & 0.134 & 0.993 & $6.4 \mathrm{E}-4$ & $2.6 \mathrm{E}-4$ & $6.5 \mathrm{E}-3$ & 0.000 \\
\hline 6 & 0.165 & 0.991 & $3.7 \mathrm{E}-4$ & $1.9 \mathrm{E}-4$ & $9.9 \mathrm{E}-3$ & -0.104 \\
\hline 7 & 0.165 & 0.993 & $5.5 \mathrm{E}-4$ & $1.9 \mathrm{E}-4$ & 7.7E-3 & -0.324 \\
\hline 8 & 0.147 & 0.990 & $6.2 \mathrm{E}-4$ & $2.1 \mathrm{E}-4$ & $1.1 \mathrm{E}-2$ & 0.305 \\
\hline 9 & 0.084 & 0.996 & $1.1 \mathrm{E}-3$ & $1.9 \mathrm{E}-4$ & $3.4 \mathrm{E}-3$ & 0.035 \\
\hline 10 & 0.128 & 0.998 & $6.3 \mathrm{E}-4$ & $2.0 \mathrm{E}-4$ & $2.2 \mathrm{E}-3$ & 0.144 \\
\hline 11 & 0.130 & 0.997 & $5.2 \mathrm{E}-4$ & $2.0 \mathrm{E}-4$ & $2.6 \mathrm{E}-3$ & -0.499 \\
\hline 12 & 0.125 & 0.997 & $5.2 \mathrm{E}-4$ & $1.7 \mathrm{E}-4$ & $2.0 \mathrm{E}-3$ & -0.055 \\
\hline $13 *$ & 0.170 & 0.992 & 3.0E-4 & $1.6 \mathrm{E}-4$ & $8.4 \mathrm{E}-3$ & 0.034 \\
\hline $14 *$ & 0.203 & 0.989 & $5.6 \mathrm{E}-4$ & $2.1 \mathrm{E}-4$ & $9.4 \mathrm{E}-3$ & -0.017 \\
\hline $15^{*}$ & 0.258 & 1.001 & 3.2E-4 & $1.5 \mathrm{E}-4$ & $-5.8 \mathrm{E}-3$ & 0.211 \\
\hline 16 & 0.127 & 0.995 & $6.3 \mathrm{E}-4$ & $4.5 \mathrm{E}-4$ & $3.5 \mathrm{E}-3$ & -0.092 \\
\hline 17 & 0.147 & 0.994 & 4.7E-4 & 4.3E-4 & $6.2 \mathrm{E}-3$ & -0.007 \\
\hline 18 & 0.077 & 0.998 & 4.3E-4 & $1.1 \mathrm{E}-4$ & $1.5 \mathrm{E}-3$ & 0.000 \\
\hline 19 & 0.132 & 0.989 & $5.0 \mathrm{E}-4$ & $2.1 \mathrm{E}-4$ & $1.0 \mathrm{E}-2$ & 0.178 \\
\hline 20 & 0.096 & 0.997 & 4.3E-4 & $9.4 \mathrm{E}-5$ & $2.6 \mathrm{E}-3$ & 0.060 \\
\hline 21 & 0.147 & 0.995 & $4.0 \mathrm{E}-4$ & $1.3 \mathrm{E}-4$ & $5.2 \mathrm{E}-3$ & -0.244 \\
\hline 22 & 0.224 & 0.992 & $6.5 \mathrm{E}-4$ & $1.8 \mathrm{E}-4$ & $6.3 \mathrm{E}-3$ & -0.030 \\
\hline 23 & 0.125 & 0.997 & $4.5 \mathrm{E}-4$ & $1.2 \mathrm{E}-4$ & $2.7 \mathrm{E}-3$ & -0.199 \\
\hline 24 & 0.095 & 0.995 & $4.2 \mathrm{E}-4$ & $-1.9 \mathrm{E}-4$ & $3.9 \mathrm{E}-3$ & -0.140 \\
\hline 25 & 0.117 & 0.995 & $3.4 \mathrm{E}-4$ & $-1.8 \mathrm{E}-3$ & $3.7 \mathrm{E}-3$ & -0.111 \\
\hline 26 & 0.103 & 0.998 & $3.1 \mathrm{E}-4$ & $-1.2 \mathrm{E}-3$ & $1.4 \mathrm{E}-3$ & 0.030 \\
\hline $27 *$ & 0.308 & 1.010 & $3.5 \mathrm{E}-4$ & $1.9 \mathrm{E}-4$ & $-1.8 \mathrm{E}-2$ & -0.060 \\
\hline Max & 0.308 & 1.010 & $1.1 \mathrm{E}-3$ & $6.5 \mathrm{E}-4$ & $1.3 \mathrm{E}-2$ & 0.305 \\
\hline Min & 0.073 & 0.989 & $3.0 \mathrm{E}-4$ & $-1.8 \mathrm{E}-3$ & $-1.8 \mathrm{E}-2$ & -0.499 \\
\hline Range & 0.234 & 0.022 & 7.6E-4 & $2.4 \mathrm{E}-3$ & $3.1 \mathrm{E}-2$ & 0.804 \\
\hline Average & 0.154 & 0.995 & $5.1 \mathrm{E}-4$ & 8.2E-5 & $3.9 \mathrm{E}-3$ & -0.048 \\
\hline $\mathrm{CV}$ & $39.9 \%$ & $0.5 \%$ & $33.3 \%$ & $579.5 \%$ & $153.3 \%$ & $-344.7 \%$ \\
\hline
\end{tabular}

Model cross validation yielded maximum and average MAE for the single node temperature models of $0.31{ }^{\circ} \mathrm{C}$ and $0.15{ }^{\circ} \mathrm{C}$, respectively. The values compare to a thermistor uncertainty of $0.2^{\circ} \mathrm{C}$. Some offices experienced illuminance values beyond the maximum value for the illuminance sensors (the offices with the higher MAE), increasing model error. Offices where the illuminance readings remained within the sensor range did not exceed a MAE of $0.22^{\circ} \mathrm{C}$. Without data from the high illuminance cases it is difficult to determine if the model error can be attributed to model for or sensor limitations. The effect of input ranges for individual offices can be observed by the relative value of the corresponding linear parameters, where outliers can be anecdotally linked to physical characteristics of the respective office. Improper sensor calibration is a current limitation within in-situ buildings research and is a source of error for any data-driven studies.

\section{Demonstrated application of IAT model}

Usage of the inverse models developed in this study is demonstrated in Figure 6, where two days of IAT prediction are shown for a typical office on unoccupied 
(July $29^{\text {th }}$ ) and occupied (July $30^{\text {th }}$ ) days. Predictions are compounded over one, three, and six time steps using weather and occupant prediction data from CanMETEO and simple logistic models to predict inputs. The use of input predictions and modelling was similar to the approaches described in the literature for MPC (Bursill et al., 2018; H Burak Gunay, O'Brien, Beausoleil-Morrison, $\&$ Bursill, 2016). The maximum IAT error for individual one (30 minute), three (1.5 hour), and six (3 hour) time step predictions for the sample room are $0.2^{\circ} \mathrm{C}, 0.5^{\circ} \mathrm{C}$, and $0.7^{\circ} \mathrm{C}$ respectively. Throughout the two-day period reasonable conformity of the model to the real IAT is observed. Further error at extended prediction periods (i.e., greater than $30 \mathrm{~min}$ ) can be attributed to inaccuracy of input predictions and is difficult to quantify within the scope of this study. For MPC applications a thorough analysis of appropriate prediction and control horizons should be executed.

The model form presented in this paper was found suitable for the offices within the building studied. Similar inputs were found suitable in other buildings and are common in new construction. Model form may vary depending on location, season, thermal mass, façade orientation, space type, and many other factors. Differential pressure (as a proxy for air flow rate) was the limiting sensor with respect to propagated error, having a bias error of $1 \%$ of full scale (correlating to $2.5 \mathrm{~Pa}$ ). The $2.5 \mathrm{~Pa}$ of differential pressure bias error typically converts to $30-100 \mathrm{~L} / \mathrm{s}$ of VAV airflow error depending on the conversion factor of the space. Airflow measurement bias error is frequently an order of magnitude higher than the other bias errors from most room level sensors (e.g., temperature, illuminance). If the bias error of the pressure transducer was halved a similar effect would be found with the propagated bias error for cooling energy (the typical measured quantity for comparing MPC algorithms in the cooling season), improving the value of energycentric studies.

Inverse modelling, as presented in this paper, has limitations relative to detailed modelling approaches. Linearization and the range of data in the training period are the most prominent. Nonlinearity can be encompassed in detailed modelling using software that has nonlinear (or pseudo-nonlinear) approaches to longwave and shortwave radiation calculations (Crawley et al., 2001). Further, cases where inputs are often beyond typical values in the training phase can yield less accurate results. In the case presented in this paper (and the proposed inverse modelling workflow) the benefits of simplicity, calculation speed, and intrinsic calibration are assumed to overcome these limitations.

Sensor logging sample resolution of 30 minutes was found to be appropriate in this study at the room level, but this will vary depending on the complexity of the system and the thermal mass present. Rooms and zones are typically thermally slow to respond (compared to plant level equipment such as boilers, chillers and air handlers) and can have a longer logging interval. Shorter sample intervals have the benefit of easing fault detection, troubleshooting, and providing additional time step selection options. An argument could be made for both longer and shorter sample intervals depending on the building characteristics and control-oriented model application. A procedure for determining the critical time step for reduced order thermal model stability is provided in the literature (Athienitis, 1994). Larger and faster systems may merit the additional accuracy achieved by increasing sampling rate by default.

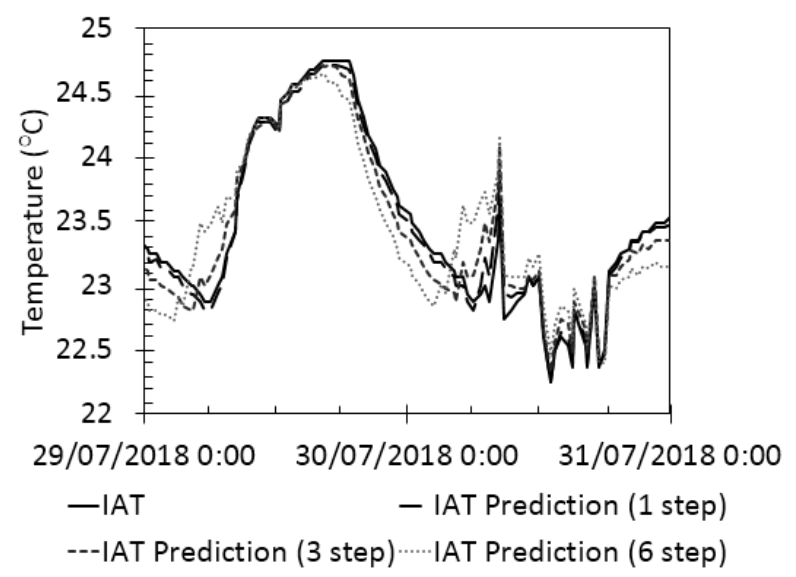

Figure 6: Two typical days from a sample room with one, three, and six time step IAT predictions.

\section{Conclusion}

This paper proposed a workflow for developing controloriented inverse models and presented the results of a case study utilizing the approach. Data acquisition, data processing, model selection, model training and validation, and model application are defined as the minimally necessary steps for inverse modelling. The proposed workflow follows a generic outline with recommendations for newer data-driven modellers on the execution of each step.

Based on the modelling results of the case study, the single node linear time-invariant state-space indoor air temperature models were determined to be suitable to predict room air temperature at the next time step for control applications. The models also proved to be effective at predicting IAT several time steps ahead with appropriate input predictions from CanMETEO weather files and occupant prediction models. In the case presented in this paper a maximum single time step MAE of $0.31^{\circ} \mathrm{C}$ was observed, with an average MAE of $0.15^{\circ} \mathrm{C}$. The simplified models were less accurate for the highly glazed rooms in this study and similar rooms may require more detailed modelling for certain applications in future work. Higher errors in highly glazed rooms is likely a result of coupling the surface temperature and air temperature into one node. The models presented are suitable for control-oriented applications due to their computational speed and relative accuracy.

Future work should include utilizing the same approach for the heating season and additional offices to determine 
if acceptable model accuracy is achieved. Additionally, the extension of these models from standalone temperature prediction to MPC calculations would further validate the approach.

\section{Acknowledgement}

This research was supported by Natural Sciences and Engineering Research Council (NSERC) of Canada, Delta Controls Inc. and Carleton University Facilities Management and Planning which are gratefully acknowledged.

\section{References}

Athienitis, A. (1994). Building thermal analysis. Electronic Book Mathcad, MathSoft, Boston, USA.

Bacher, P., \& Madsen, H. (2011). Identifying suitable models for the heat dynamics of buildings. Energy and Buildings, 43(7), 1511-1522.

Bursill, J., O’Brien, W., \& Beausoleil-Morrison, I. (2018). Experimental application of classification learning to generate simplified model predictive controls for a shared office heating system. Science and Technology for the Built Environment(just-accepted), 1-38.

Candanedo, J., \& Athienitis, A. (2011). Predictive control of radiant floor heating and solar-source heat pump operation in a solar house. $H V A C \& R$ Research, 17(3), 235-256. doi: 10.1080/10789669.2011.568319

Charpenay, V., Käbisch, S., Anicic, D., \& Kosch, H. (2015). An ontology design pattern for iot device tagging systems. Paper presented at the Internet of Things (IOT), 2015 5th International Conference on the.

Crawley, D. B., Lawrie, L. K., Winkelmann, F. C., Buhl, W. F., Huang, Y. J., Pedersen, C. O., ... Witte, M. J. (2001). EnergyPlus: creating a newgeneration building energy simulation program. Energy and Buildings, 33(4), 319-331.

Dibowski, H., Vass, J., Holub, O., \& Rojíček, J. (2016). Automatic setup of fault detection algorithms in building and home automation. Paper presented at the Emerging Technologies and Factory Automation (ETFA), 2016 IEEE 21st International Conference on

Dong, B., O'Neill, Z., \& Li, Z. (2014). A BIM-enabled information infrastructure for building energy Fault Detection and Diagnostics. Automation in construction, 44, 197-211.

Froisy, J. B. (2006). Model predictive control—building a bridge between theory and practice. Computers \& chemical engineering, 30(10-12), 1426-1435.

Gouda, M., Danaher, S., \& Underwood, C. (2002). Building thermal model reduction using nonlinear constrained optimization. Building and environment, 37(12), 1255-1265.
Gunay, B., O’Brien, W., \& Beausoleil-Morrison, I. (2016). Control-oriented inverse modeling of the thermal characteristics in an office. Science and Technology for the Built Environment, 22(5), 586-605. doi: 10.1080/23744731.2016.1175893

Gunay, H. B., Bursill, J., Huchuk, B., O'Brien, W., \& Beausoleil-Morrison, I. (2014). Shortestprediction-horizon model-based predictive control for individual offices. Building and environment, 82, 408-419. doi: 10.1016/j.buildenv.2014.09.011

Gunay, H. B., O'Brien, W., Beausoleil-Morrison, I., \& Bursill, J. (2016). Implementation of an adaptive occupancy and building learning temperature setback algorithm. ASHRAE Transactions, 122, 179.

Joe, J., \& Karava, P. (2016). Agent-based system identification for control-oriented building models. Journal of Building Performance Simulation, 10(2), 183-204. doi: 10.1080/19401493.2016.1212272

Kim, D., \& Braun, J. E. (2014). A general approach for generating reduced-order models for large multi-zone buildings. Journal of Building Performance Simulation, 8(6), 435-448. doi: 10.1080/19401493.2014.977952

Ljung, L. (1995). System Identification Toolbox for use with MATLAB.

Salsbury, T. I. (2005). A survey of control technologies in the building automation industry. IFAC Proceedings Volumes, 38(1), 90-100.

Shahin, M. A., Maier, H. R., \& Jaksa, M. B. (2004). Data division for developing neural networks applied to geotechnical engineering. Journal of Computing in Civil Engineering, 18(2), 105114.

Shi, Z., \& O'Brien, W. (2018). Building energy model reduction using model-cluster-reduce pipeline. Journal of Building Performance Simulation, 11(5), 553-567.

Wang, L. (2009). Model predictive control system design and implementation using MATLAB®: Springer Science \& Business Media.

Yang, J., Rivard, H., \& Zmeureanu, R. (2005). On-line building energy prediction using adaptive artificial neural networks. Energy and Buildings, 37(12), 1250-1259.

Zhao, J., Lam, K. P., Ydstie, B. E., \& Karaguzel, O. T. (2015). EnergyPlus model-based predictive control within design-build-operate energy information modelling infrastructure. Journal of Building Performance Simulation, 8(3), 121134.

Zhou, N., Pierre, J. W., \& Hauer, J. F. (2006). Initial results in power system identification from injected probing signals using a subspace method. IEEE Transactions on Power Systems, 21(3), 1296-1302. 